Methods Human MSC were isolated from healthy controls Comparisons were made between podoplanin positive and negative MSC. MSC migration across 8 um pore filters following treatment with anti-siRNA podoplanin or Rho GTPases inhibitors was assessed. MSC-platelet interactions were assessed by culturing MSC on the basal surface of $3 \mathrm{um}$ pore filters and perfusing fluorescently labelled platelets in whole blood over the apical surface. In some cases, the apical surface of the filter was pre-coated with EC, forming an EC-MSC coculture, prior to platelet perfusion.

Results Expression of podoplanin significantly enhanced the migration of MSC compared to MSC lacking podoplanin. Rac-1 inhibition altered the membrane localisation of podoplanin and in turn significantly reduced MSC migration. Blocking Rac-1 activity had no effect on the migration of MSC lacking podoplanin, indicating it was responsible for regulation of migration through podoplanin. When podoplanin-expressing MSC were seeded on the basal surface of a porous filter, they were able to capture platelets perfused over the uncoated apical surface and induce platelet aggregation. Similar microthrombi were observed when EC were co-cultured on the apical surface. Confocal imaging shows podoplanin-expressing MSC extending processes into the EC layer, which could interact with circulating platelets. In both models, platelet aggregation induced by podoplanin-expressing MSC was inhibited by recombinant soluble CLEC-2.

Conclusions Podoplanin enhances the migratory capacity of tissue-resident MSC enabling them to move more rapidly within the rheumatoid joint. Moreover, podoplanin allows MSC to interact with both circulating and tissue platelets to elicit either protective or pathogenic responses.

Acknowledgements This work was funded by an Versus Arthritis Career Development Fellowship, a MRC-funded PhD studentship and BHF Grant.

Disclosure of Interest None declared.

\section{P119 EPIGENETIC REGULATION OF WNT SIGNALING IN SYSTEMIC SCLEROSIS VIA SFRP1}

S O'reilly*. Life Sciences, Northumbria University, Newcastle Upon Tyne, UK

\subsection{6/annrheumdis-2018-EWRR2019.107}

Career situation of first and presenting author Young investigator.

Introduction Systemic sclerosis is an autoimmune connective tissue disease. The disease is characterised by inflammation and fibrosis. The main cell type is an activated fibroblast called a myofibroblast that secretes a panopoly of extracellular matrix molecules lieading to the skin and lung fibrosis. The mechanism(s) that lead to the myofibroblast generation are unknown. In recent times epigenetic regulation has been shown to play a major role in generation of myofiibroblasts in systemic sclerosis and other fibrotic diseases. Epigenetics is defined as a change in gene expression without a change in the DNA bases. This is mainly facilitated by DNA methylatedl the addition of a methyl group onto cytosine and microRNAs. Wnt is a conserved signaling pathway that is needed for organ formation and is known to be elevated in systemic sclerosis. Enhanced Wnt can lead to fibrosis through beta catenin and extracelluar antagonists regulate the threshold of Wnt signalling. sFRP1 is an extracellular
Wnt inhibitor that we have previously demonstrated to be involved in systemic sclerosis.

Objectives To determine the epigenetic regulation of sFRP1 in systemic sclerosis.

Methods Diffuse systemic sclerosis and healthy control serum was collected from 10 patients and controls. sFRP1 was measured with a standard ELISA method using a commercial ELISA. qPCR was perfromed with SSc fibroblasts or control fibroblasts using Taqman specific kits for miR27a3p and RNU44. Data was normalised to RNU44 and shown as fold change to controls. Transfection of microRNA mimics was performed using $100 \mathrm{nM}$ of miR27 mimics or matched concentration of scramble after 48 hours post transfection media was collected and the cells lysed in RIPA buffer and lysates subjected to western blotting.

Results We found reduced levels of sFRP1 in systemic sclerosis sera compared to healthy controls $(n=10)$. Using taqman PCR we also found elevated levels of microRNA27a3p in systemic sclerosis fibroblasts. Using software to predict targets it was identified that sFRP1 is a direct target of microRNA27a3p. We could demonstrate after transfection of microRNA27a3p into healthy dermal fibroblasts compared to scramble controls that the levels of the target sFRP1 was reduced and elevated levels of collagen 1 and beta catenin was present. This suggest that sFRP1 is a direct target leading to upregulation of Wnt signaling. We also found reuced levels of anti-fibrotic PPARgamma also after transfection. siRNA knockdown of sFRP1 using small interfering RNA leads to upregulation of collagen and Axin2 and lactate elevation.

Conclusions sFRP1 is regulated by miR27a2p in systemic sclerosis. MiR27a3p can also regulate PPAR gamma. Enhanced Wnt signaling is associated with metabolic alterations. Disclosure of Interest None declared.

\section{P120 IL-23 RECEPTOR SIGNALING IS IMPORTANT DURING PHYSIOLOGICAL BONE REMODELING AND RADIAL BONE GROWTH THROUGH REGULATION OF OSTEOBLAST DIFFERENTIATION}

${ }^{1} \mathrm{~W}$ Razawy ${ }^{*},{ }^{2} \mathrm{M}$ Schreuders-koedam, ${ }^{1} \mathrm{P}$ Asmawidjaja, ${ }^{1} \mathrm{~A}$ Mus, ${ }^{1} \mathrm{H}$ Den Braanker, ${ }^{3} \mathrm{M}$ Oukka, ${ }^{4} \mathrm{~V}$ Kuchroo, ${ }^{2} \mathrm{~B}$ Van der Eerden, ${ }^{1} \mathrm{E}$ Lubberts. ${ }^{1}$ Rheumatology; ${ }^{2}$ Internal Medicine, Erasmus Medical Centre, Rotterdam, Netherlands; ${ }^{3}$ Pediatrics, Seattle Children's Research Institute, Seattle; ${ }^{4}$ Centre for Neurologic Diseases, Harvard Institute of Medicine, Boston, USA

\subsection{6/annrheumdis-2018-EWRR2019.108}

Career situation of first and presenting author Student for a master or a $\mathrm{PhD}$.

Introduction In mice, systemic exposure of IL-23 induces chronic arthritis, increased osteoclast differentiation and systemic bone loss. However, the role of IL-23R signaling during physiological bone remodeling is not fully elucidated.

Objectives To examine the role of IL-23R signaling during physiological bone remodeling.

Methods Femurs of naïve 7-, 12- and 26-week-old IL-23R ${ }^{\mathrm{GFP} /}$ GFP (IL-23R $\mathrm{R}^{-/}$) and IL-23R $\mathrm{R}^{+/+}$(WT) littermate mice were used for micro-CT analysis of the bone and a three-point bending test for bone strength. Bone marrow (BM) cells were either cultured towards osteoclasts with M-CSF and RANKL or were cultured towards osteoblasts with $\beta$-glycerophosphate and vitamin C. Osteoclast differentiation and activity were assessed 